\title{
La Guerra Civil en El Casar de Escalona. Del paseo militar a la resistencia planificada (septiembre 1936)
}

\author{
juan Carlos Collado Jiménez
}
The Spanish Civil War in El Casar de Escalona. From military occupation to planified resistance (September 1936)

\begin{abstract}
RESUMEN
La pretensión de este artículo es analizar los hechos que acontecieron en la localidad de El Casar de Escalona durante el verano de 1936, principalmente en septiembre. En su recorrido por Andalucia y Extremadura, el avance del ejército de África fue un "paseo militar"; sin embargo, tras la "conquista" de Talavera de la Reina, el gobierno de la República organizó varias formas de resistencia en el eje Cazalegas-Maqueda y una de ellas, se colocó frente a El Casar de Escalona.
\end{abstract}

PALABRAS CLAVE:

Guerra Civil; Ejército de África; Milicias Republicanas; "Marcha hacia Madrid"; "Columna Castejón".

\begin{abstract}
This article aspires to analyze the events that took place in the locality of El Casar de Escalona during the summer and particularly September of 1936. The advance of the African troops through Andalucia and Extremadura was a "military promenade" although after the conquest of Talavera de la Reina, the government of the Republic organized various patterns of resistance in the Cazalegas-Maqueda axis. One of them was placed in front of El Casar de Escalona.
\end{abstract}

KEY WORDS:

Civil War; African Army; Republican troops; "March towards Madrid"; "Columna Castejón".

\section{INTRODUCCIÓN}

El microcosmos elegido para la investigación ha sido la guerra civil española en la localidad de El Casar de Escalona, situada al norte de la provincia de Toledo.

1 El presente trabajo se basa en un extracto de una investigación más amplia, acotada cronológicamente al año 1936, que se adentra brevemente en los antecedentes de la contienda, hace especial hincapié en la "guerra de las columnas" y finalmente refleja alguno de sus efectos y consecuencias. 
Mediante la historia política podemos conocer la vida de un país, una nación o un pueblo; gracias a ella, podemos acercarnos a los sucesos de marcos gigantescos, pero también nos sirve para explicar los hechos históricos de una ciudad o de un pequeño pueblo. Esto no significa que los sucesos del lugar que estudiemos sean independientes de otros del periodo, por ello, pasaremos de lo particular a lo general y viceversa. La pretensión de este artículo es analizar, lo más objetivamente posible, los hechos acaecidos en este pueblo una vez iniciado el enfrentamiento armado, principalmente en septiembre, y algunas de sus consecuencias.

Tanto los testimonios orales como las crónicas sobre la historia de la villa hablan de fuertes combates por su ocupación en los días 15 y 17 de septiembre de 1936. Se sabe que las perdidas humanas fueron muy elevadas. ¿Por qué este artículo? Primero, por la curiosidad intelectual sobre lo sucedido en el lugar, no exento de contradicciones, y también por el vacío bibliográfico existente sobre el conflicto bélico en una zona, el eje Cazalegas-Maqueda, vital para el devenir de «la marcha hacia Madrid». Desde luego, un trabajo de esie tipo centrado en El Casar de Escalona es inédito. No obstante, esperamos una avalancha de monografías sobre la guerra civil en lo que resta del año 2005 y en el 2006, coincidiendo con el setenta aniversario del inicio del conflicto.

Hemos dividido este artículo en dos apartados. El primero, explica la primera fase de la guerra, y dentro de ésta el ciclo denominado "guerra de las columnas" o «fase miliciana de la guerra» caracterizado por el "paseo militar" del ejército nacional por tierras de Andalucía y Extremadura hasta su entrada en la provincia de Toledo el 28 de agosto de 1936. El segundo apartado, base del trabajo, está destinado a analizar la organizada resistencia republicana que ralentizó sobremanera la ocupación de los pueblos del sector Talavera-Santa Olalla en el camino de la «Columna Madrid", con especial dedicación a las fortificaciones del eje Cazalegas-Maqueda (del 10 al 21 de septiembre de 1936) y a los fuertes combates del 15 de septiembre que culminaron en el "martes sangriento» de El Casar de Escalona.

Aspiramos a buscar la respuesta a muchos interrogantes. Una vez levantado en Marruecos, el ejército de África se encontró con su principal problema y su más difícil resolución ¿cómo llegar a la península? ¿triunfó el alzamiento en los pueblos de la provincia de Toledo? ¿en qué consistió la "guerra de las columnas"? Hasta su entrada en la provincia fue una "guerra relámpago" ¿qué cambió en esta fase bélica, en la que se vio involucrado El Casar? ¿Es cierto que se rechazó a la "Columna Castejón frente al pueblo?... Con lo que tenemos, intentaremos acercarnos a la verdad.

\section{LAS CAMPAÑAS MILITARES DEL VERANO DE 1936}

\subsection{El comienzo de la contienda}

El viernes 17 de julio de 1936 se produjo en la guarnición de Melilla el primer acto de insurrección militar contra la República española, seguido de otros de 
parecido calibre en los cuarteles de la peninsula favorables a los golpistas. Ante el fracaso como levantamiento fulminante, el lunes día 20 salieron a combatir las primeras columnas y el conflicto se alargó en el tiempo nada menos que hasta el 1 de abril de 1939. La Castilla del sur -incluida Madrid-se mostró fiel al gobierno con la excepción de algunos enclaves rebeldes². La insurrección militar encontró enseguida partidarios. Respecto a las actuales provincias de Castilla-La Mancha, así sucedió en las capitales de Toledo, Albacete y Guadalajara, aunque sofocada en las dos últimas. En general, siguiendo a Ruiz $\mathrm{Alonso}^{3}$, en los pueblos de la provincia de Toledo los conspiradores no lograron sus propósitos ante la ausencia de la Guardia civil -que los abandonó para dirigirse a la capital toledana-y en los municipios que se desencadenó, no duró más de dos o tres dias. En El Casar de Escalona, como en Calera y Chozas, Carmena, Escalonilla, Pelahustán o La Puebla de Montalbán, entre otros lugares relativamente cercanos, las fuerzas populares triunfaron rápidamente haciendo demostración y acciones de fuerza de distinta intensidad según la localidad.

Una vez fracasado el golpe militar los sublevados aspiraban a pasar el ejército africano a la península. Ya el 20 de julio cruzaron en avión el Estrecho cuarenta legionarios de la quinta Bandera del Tercio con el comandante Castejón y, progresivamente, continuaron llegando el resto por distintos medios. Entre el 27 y 28 de julio, gracias a la ayuda de aviones italianos y alemanes de gran capacidad de transporte, en poco más de una semana fue posible el paso de 14.000 hombres con todos sus pertrechos en un impresionante puente aéreo. Con la visita que el 2 de agosto hizo al puerto de Ceuta el acorazado alemán Deutschland se facilitó el acceso definitivo de los convoyes con el resto del ejército de Marruecos ${ }^{4}$. Ante la importancia del hecho, las autoridades republicanas intentaron ocultar durante el mayor tiempo posible la entrada, así como la presencia de "moros» en la península.

La guerra fue principalmente una lucha entre españoles pero el conflicto no hubiera sido muy largo de no haberse internacionalizado: la política de «No intervención" en España resultó totalmente falsa, pues nadie permaneció neutral. Los nacionales contaron con la intervención alemana e italiana - mediante la flota, hombres, material y armas-el aporte de soldados de Marruecos y la ayuda del Portugal salazarista, vía de entrada de suministros de sus ejércitos. En el otro lado, la colaboración que recibió la República podemos considerarla muy inferior: la soviética, la muy escasa llegada desde México y la participación de las brigadas internacionales. En todo caso, ante el fracaso como golpe militar, gracias a la ayuda alemana e italiana, los nacionales consiguieron introducir el ejército en la península. Este logro, de gran trascendencia, se ocultó por el alto mando de la República.

\footnotetext{
2 ARóstegul, J., "La Guerra Civil, 1936-1939. La ruptura democrática" en Historia de España. Madrid, Historia 16 Temas de Hoy, n. ${ }^{\circ} 27,1996$, pp. 32-33.

${ }^{3}$ Ruiz Alonso, J. M., La Guerra Civil en la provincia de Toledo. Utopía, conflicto y poder en el sur del Tajo (1936-1939). Ciudad Real, Biblioteca Añil, 2004, tomo I, pp. 191-192.

"TAmames, R., "La República. La era de Franco", Historia de España Alfaguara. Madrid, Alianza Editorial, n. ${ }^{\circ}$ VII, pp. $243-244$.
} 


\subsection{La primera fase de la guerra}

En la guerra civil podemos distinguir varios ciclos. El primero, transcurrió desde el inicio de las operaciones militares en campo abierto hasta marzo de 1937 con la batalla de Guadalajara y último intento de los rebeldes por conquistar Madrid. Dentro de este periodo, El Casar de Escalona fue ocupado en su fase inicial, al estilo colonial.

La guerra se modernizó paulatinamente pero en sus comienzos presentó rasgos arcaicos, desfasados no solamente para la época, sino incluso en comparación con las técnicas de la Primera Guerra Mundial. Las agrupaciones principales se basaron en las columnas y las tropas, de ahí la famosa denominación de "la guerra de las columnas" o "fase miliciana de la guerra» 5 . Las fuerzas militares nacionales mostraron desde el principio un férreo control militar; sin embargo, la República se quedó inicialmente sin ejército -éste quedó disuelto-y, aunque bajo mando castrense, sus primeros efectivos eran un conglomerado de fuerzas regulares, fuerzas del orden público y milicianos agrupados en unidades creadas por partidos y sindicatos. A partir de septiembre y octubre se llevaron a cabo medidas para la militarización de las milicias y la creación de un Ejército Popular Regular.

A finales de julio, la mayoría de las provincias de la reciente Comunidad Autónoma de Castilla-La Mancha estaban bajo el mando republicano, con la excepción del Alcázar de Toledo y una franja del noreste de Guadalajara. A partir del 5 de agosto, una vez conseguido el paso del ejército de África a la península por el estrecho de Gibraltar, las columnas avanzaron hacia el norte no por Andalucia como hubiese sido lo más lógico, sino pegadas a la frontera portuguesa, con el apoyo del país vecino, de Alemania y de Italia. El ejército nacional llegó a Sevilla el 7 de agosto e inició la marcha hacia Mérida y Badajoz -el 11 de agosto ocuparon Mérida y el 14 Badajoz - y a continuación, el día 15, se dirigieron por la carretera general Cáceres-Madrid hacia Trujillo y Navalmoral de la Mata antes de adentrarse por el valle del Tajo en dirección a Toledo .

En definitiva, a partir del 28 de julio de 1936, con la llegada de los primeros aviones alemanes a Marruecos en ayuda de Franco se abrió la fase dinámica de la guerra, denominada «la guerra de las columnas", la única que, a pesar de sus procedimientos anticuados, funcionó como campaña relámpago por el rápido avance del ejército del sur a través de Andalucía, Extremadura - escasamente pobladay el valle del Tajo $\sin$ baluartes defensivos.

5 La columna no era otra cosa que una forma de agrupación militar con prácticas guerreras heredadas de las contiendas coloniales precedentes; normalmente se las nombraba por el nombre de su jefe, por ejemplo "Columna Castejón».

6 Aróstegui, J., "La Guerra Civil», Cuadernos Historia 16. Madrid, Historia 16, n. ${ }^{\circ}$ 2, 1985, p. 18. 


\subsection{La llegada del conflicto a la provincia de Toledo}

A finales del verano estaban en la peninsula 20.000 "moros" y legionarios organizados en columnas de 500 a 1.000 hombres bajo el mando de los siguientes oficiales: el general Varela, los coroneles Yagüe y Carlos Asensio, los tenientes coroneles Barrón, Delgado y Tella, los comandantes Castejón y Mizzian, que servian al Tercio de extranjeros creados por Franco. La denominada "Columna Madrid" estaba formada por las columnas africanas de Asensio y Castejón; posteriormente se unió también la de Tella. Con estos efectivos, el día 28 de agosto de 1936, las tropas del ejército de África se adentraron en la provincia de Toledo por el municipio de Calzada de Oropesa y las Ventas de San Julián. La Columna, al mando primero de Yagüe y después de Varela, atravesó la provincia de Toledo de oeste a este en dos meses y finalizó su travesía el 26 de octubre en El Viso de San Juan; sin embargo, los municipios situados al sur del rio Tajo no se ocuparon hasta finales del mes de marzo de 1939, al final ya de la guerra civil?.

El periodista y escritor Franz Borkenau realizó varios viajes a España durante el conflicto y se convirtió en un cronista de excepción en esta fase decisiva de la guerra. Recorrió los pueblos del occidente toledano entre el 31 de agosto y el 1 de septiembre, cuando las tropas de Yagüe ocuparon Oropesa. Resulta cuando menos curiosa su descripción de las poblaciones que encuentra en su trayecto a Talavera. En cuanto al nivel de vida las diferencias son notables respecto a Levante y Cataluña, su anterior viaje por la España en guerra ( y, en vez de fruta y verduras, aquí se cultiva trigo»). Le llamó la atención que en las tierras de Castilla La Nueva - tenemos el ejemplo de El Casar de Escalona- continuaron ejerciendo su autoridad los ayuntamientos junto con los comités políticos. Además percibió que, a diferencia de otras zonas, aqui dominaron los socialistas y no los anarquistas. A pesar de la extrema pobreza del sur castellano, o como consecuencia de ella, los campesinos defendían con entusiasmo la causa republicana, quizá porque el problema de la tierra estaba aún sin resolver ${ }^{8}$.

Gabriel Jackson describe detalladamente la manera en que se llevó a cabo la ocupación de los pueblos en esta "fase miliciana de la guerra"9. Las columnas viajaban en camiones que solían detenerse a una distancia prudencial de cada pueblo para que los hombres avanzaran a pie. Si habia indicios de resistencia, la artillería ligera bombardeaba los lugares que pudiesen ser reductos (muros o edificios de piedra). A continuación, se tomaba la localidad en una carga a la bayoneta y con unos altavoces se ordenaba que se abriesen las puertas de las casas y se desplegaran banderas blancas. Los que eran sorprendidos con armas $\longrightarrow$ con el hombro magullado por el retroceso de un fusil o las marcas de las mochilas- eran

7 Sabin, J. M., “Toledo y la guerra» en OrTiz Heras, M., (Coord.), La guerra civil en Castilla-La Mancha. Toledo, Biblioteca Añil, 2000, pp. 141-146.

- Borkenau, F., El reñidero español. Barcelona, Ediciones Península, 2001, pp. 168 y 172.

9 Jackson, G., La República Española y la Guerra civil. Madrid, Critica, 2005, pp. 241-242. 
fusilados y los milicianos que huían por las carreteras caían ametrallados por armamento de este tipo colocado en las mismas. En cualquier caso, la represión fue superior en los objetivos que ofrecieron mayor resistencia, como en el caso de $\mathrm{El}$ Casar de Escalona semanas después. Lo normal es que quedase un pelotón atrás para asegurar el pueblo y las comunicaciones mientras la agrupación proseguía su ruta hacia el norte.

De acuerdo con la forma reseñada ${ }^{10}$, el avance de las columnas del ejército del Sur desde Sevilla a Mérida parecía un "paseo militar", dada la escasa resistencia de los campesinos que encontraron a su paso - armados con escopetas de caza, algunos fusiles- y la esporádica protección aérea que el Gobierno republicano enviaba desde Cuatro Vientos y Getafe. La excepción se producía cuando algunos milicianos se atrincheraban en algún edificio, dispuestos a morir. La Legión y los Regulares, dotados de artillería, carros de combate y con apoyo aéreo se limitaron a hacer la guerra que habían practicado contra los rifeños en las campañas de Marruecos. En todo caso, si solamente emplearon dieciséis días para recorrer los 218 Kilómetros que separan Mérida de Talavera y tres para conquistar esta ciudad (a razón de $11,5 \mathrm{Km} / \mathrm{dia}$ ), las cosas comenzaron a cambiar y, como veremos más adelante, para recorrer los 83 Kilómetros de distancia entre Talavera y Torrijos, tardaron nada menos que veintitrés días (a una media de 3,6 Km/dia). La caída de Talavera de la Reina tuvo consecuencias políticas fulminantes - la dimisión de José Giral- y el impacto provocado llevó a los responsables militares a replantearse la estrategia.

En síntesis, en su camino por Andalucía y Extremadura, la marcha del ejército de África fue un «paseo militar». El 28 de agosto llegaron a la provincia de Toledo pero, tras la toma de Talavera, los acontecimientos empezaron a evolucionar.

\section{EL CAMBIO DE ESTRATEGIA: LA RESISTENCIA PLANIFICADA}

\subsection{La defensa del norte provincial}

La forma de ocupación de los pueblos descrita anteriormente continuó al entrar en la provincia de Toledo pero la impotencia inicial de las milicias de la República comenzó a tornarse más eficaz a comienzos de septiembre, con la llegada al valle del Tajo y principalmente al Alberche, merced a las nuevas medidas adoptadas por el gobierno de Largo Caballero y el mando militar del teniente coronel republicano Asensio Torrado. Si bien el avance principal de la "Columna Madrid» siguió la línea de la carretera de Extremadura, se complementó con incursiones o movimientos secundarios. La pretensión principal no era otra que enlazar con el ejército del Norte, por ello, el 8 de septiembre, partieron de Oropesa la "Columna Delgado" y de Talavera la de Tella con el objeto de apoderarse de Arenas de San Pedro y co-

\footnotetext{
10 Ruiz Alonso, J. M., Op. Cit., pp. 305-307.
} 
nectar con una columna de Caballería de dicho ejército, operación finalizada con éxito el día 9 con la unión de las tres columnas ${ }^{11}$.

El mismo día 8, Yagüe propuso la reorganización de las columnas y agrupaciones que quedarían dirigidas por los tenientes coroneles Asensio y Barrón, comandante Castejón y teniente coronel Delgado Serrano. Franco aprobó el plan con algunas variaciones en la composición de las mismas y dejó a la Columna de Delgado en reserva. Por su importancia, la reestructuración apareció reflejada en el parte de guerra del ejército nacional de ese día. Los partes de guerra son el relato oficial de las operaciones de uno y otro bando; los del ejército nacional dividían la información en "Ejércitos" y los republicanos en "Frentes" y el 12 de septiembre nació el Frente del Centro, con Toledo incluido (Talavera de la Reina continuó en el de Extremadura) ${ }^{12}$.

Por su parte, ante el desastre de Talavera, las milicias republicanas organizaron diversos modos de resistencia ${ }^{13}$. De esta forma, podemos distinguir tres situaciones para definir la defensa de esta zona provincial. En primer lugar, resistencias apoyadas en los núcleos de población (Oropesa, Calera, Talavera, Maqueda, Toledo, Escalona y Santa Cruz de Retamar). En segundo lugar, contraataques sobre Talavera, Maqueda, Toledo, lllescas y Seseña y por último, el intento de establecer un frente defensivo escalonado entre Cazalegas y Maqueda, con El Casar de Escalona de por medio. Éste, elaborado por Asensio Torrado ralentizó la campaña militar de la "Columna Madrid" y causó numerosas bajas al bando nacional.

En definitiva, en el mes de septiembre el ejército nacional proseguía avanzando en la marcha hacia Madrid y su táctica de incursiones complementarias mientras que, los mandos militares de las milicias republicanas organizaron varias formas de resistencia, una de ellas, basada en baluartes defensivos se colocó frente a El Casar, entre Cazalegas y la estratégica población de Maqueda. Se planificaron métodos militares, se concentraron efectivos y se pasó sutilmente a la acción.

\subsection{El eje Cazalegas-Maqueda}

La entrada de Largo Caballero en el gobierno de la República supuso un cambio sustancial en el teatro de operaciones. Mediante una Directiva de 11 de

11 Documentación Nacional. Operaciones sobre Madrid: «Avance Nacional de Talavera a Maqueda del 3 al 21 (Varias Columnas)». Archivo General Militar de Ávila (AGMA). Legajo 2/18.

12 DNOM: “Organización de la Columna de Madrid (Yagüe)”, Talavera, 8 de septiembre de 1936. AGMA. Legajo 3/1.1. Idem, Cáceres, 10 de septiembre de 1936. Legajo 3/1.1. Véase en este sentido también La Marcha sobre Madrid, Servicio Histórico Militar. Madrid, Editorial San Martín, 1982, p. 175. Así se recoge igualmente en el "parte de guerra» del 10 de septiembre del 36: Partes Oficiales de guerra. 1936-1939, Ejército Nacional. Madrid, Librería Editorial San Martín, 1977, tomo I, p. 36. En la otra órbita, revísese: Partes Oficiales de guerra. 1936-1939, Ejército de la República, Servicio Histórico Militar. Madrid, Libreria Editorial San Martín, 1978, tomo II, p. 46.

13 Ruiz Alonso, J.M., Op. Cit., II: pp. 520-521. 
septiembre se dividió el Centro en cinco sectores: Guadalajara, Somosierra, Guadarrama, Extremadura y Toledo. Al mando de cada sector, con columnas de más de 4.000 hombres, se colocó un jefe militar, auxiliado por un Estado Mayor y al frente de los Sectores de Extremadura ("del Tajo") y Toledo, cuyos efectivos crecian de forma imparable, quedaron el ya general Asensio Torrado y el capitán Antonio Rubert. La decisión de guarnecer el terreno se debió al primero y por su iniciativa se llevaron a cabo numerosas obras de fortificación ante los pueblos de Cazalegas, Los Cerralbos, Illán de Vacas, El Bravo, El Casar de Escalona y Maqueda. A este dispositivo militar, ideado por el general Masquelet -jefe del Cuartel Militar del Presidente de la República el 18 de julio-y dirigido por los ingenieros toledanos Rafael Enrique Ramírez Cardeñas y Manuel Díaz-Marta Pinilla, se le dio una especial trascendencia en esta etapa del conflicto, hasta tal punto que el Presidente Azaña visitó Maqueda durante los combates ${ }^{14}$.

De acuerdo con las "Ordenes de Operaciones" del ejército nacional -en donde podemos comprobar que por un tiempo la «Columna Madrid» pasó a denominarse Ejército Expedicionario- observamos que ya el día 10 de septiembre se preveía el avance de la "Columna Asensio" para ocupar Cazalegas, Lucillos, Otero y El Casar y el 11 se instaba a la de Castejón a proseguir de Navamorcuende a Pelahustán y de Hinojosa a El Real de San Vicente. Sin embargo, la primera línea de la defensa colocada en Cazalegas, con alambradas y trincheras hasta el río Tajo, impidió controlar este pueblo hasta pasados dos días - -11 y 12 de septiembre - con más de 120 bajas del ejército de Marruecos. Yagüe pretendía acorralar a los republicanos pero, como éstos estaban apoyados en el río Tajo y en el Alberche, acudió a la ruptura frontal, propósito que no consiguió hasta el día 11 de septiembre en las proximidades de Cazalegas ${ }^{15}$.

Paralelamente, en su línea de incursiones complementarias, el día 11 de septiembre el ejército nacionalista ocupó Navamorcuende y el 12, El Real de San Vicente. Los partes de guerra nacionales se hacían eco de las conquistas. Una vez tomada Cazalegas, en la madrugada del mismo día 12, comenzó el avance de la Agrupación Castejón en dirección a Pelahustán y la de Asensio a El Casar de Escalona, ya como objetivo militar; en su camino - siempre según la versión nacional- hicieron 360 muertos y cogieron armamento y diverso material del enemigo. Los partes republicanos de esa jornada, como siempre muy ambigüos, únicamente reflejaron movimientos de tropas leales y del cumplimiento de los objetivos

14 El día 10 de septiembre se incorporaron desde Barcelona la Columna López-Tienda o «Libertad» y el 19 tercio de la Guardia civil barcelonesa del coronel Escobar que se distribuyó entre las Columnas Del Rosal y López-Tienda. Las de este último se situaron hasta el Alberche y las de Burillo en la zona de Maqueda. A estos efectos resulta de especial interés Dominación Roja. Columnas varias: «Columna López-Tienda” y “Columna Rosal». AGMA. Referencia $966 / 786$ y $967 / 787$. Así aparece también reflejado en La Marcha sobre Madrid, Op. Cit., pp. 171 y 174.

15 DNOM: “Ordenes de Operaciones (Ejército Expedicionario)», Cáceres, 10 y 11 de septiembre 1936. AGMA. Legajo 2/21.2, pp. 1-2 y 7 . Es ilustrativo el relato presentado en La Marcha sobre Madrid, Op. Cit., p. 176. Véase también en Ruiz Alonso, J. M., Op. Cit., II: p. 321. 
ordenados. El parte nacional del día 13 no hacia referencia a las operaciones desarrolladas en el eje, quizá difuminadas por la toma de San Sebastián en el País Vasco. El parte republicano sí mencionaba bombardeos en la carretera de Cazalegas que hicieron huir a un "contingente faccioso", pero lo cierto es que, como ya hemos visto, el citado pueblo lo perdió la República el 12 de septiembre ${ }^{16}$.

El ejército rebelde en su estrategia de envolver a las milicias republicanas por el norte y por el sur contó con las columnas de Barrón, Delgado y Castejón. EI día 14 de septiembre la localidad de El Casar se convirtió en uno de los objetivos particulares de los bombardeos de la aviación antes de proceder a su ocupación, al igual que Hormigos, Lucillos, Otero y Santa Olalla. En ese dia cayeron dos bombas en sendos domicilios particulares del pueblo ${ }^{17}$.

Franco pretendía que la "Columna Castejón» se concentrase en la línea Castillo de Bayuela-Cardiel de los Montes para pasar de noche el río Alberche por el vado de Cardiel y atacar al amanecer del día 15 las posiciones milicianas de El Casar de Escalona y Hormigos. Por su parte, Delgado Serrano ocuparia Lucillos, Otero y Santa Olalla. Si todo salía según lo previsto, quedarían guarniciones en Lucillos, Otero y El Casar. Pero el ejército nacionalista encontró una fuerte resistencia y varias líneas de trincheras con alambradas a unos dos kilómetros en dirección norte-sur, perpendicular a su marcha ${ }^{18}$. En suma, el cambio de táctica del mando republicano complicó y dificultó claramente el avance nacional, causándole numerosas bajas (como en el asalto a Cazalegas). El Casar de Escalona sufrió esos días los bombardeos previos que anunciaban su inminente «liberación».

\subsection{El «martes sangriento»}

La segunda línea de contención republicana en el eje se colocó en El Casar de Escalona. Dejando a un lado las construcciones situadas a varios kilómetros del pueblo, la defensa frente al casco urbano se basó en una pequeña fortificación de carros y en un reguero, utilizado como trinchera, desde "Los Charcones" hasta el «Camino de la Fuente".

${ }^{16}$ Tomado de los partes de guerra de los dias 11, 12 y 13-9-36: Partes_Ejército Nacional, pp. 37-38 y Partes ... Ejército Republicano, pp. 47-50. Para una información más pormenorizada consúltese DNOM: “Avance Nacional de Talavera a Maqueda del 3 al 21 (Varias Columnas)». AGMA. Legajo $2 / 18$.

17 DNOM: “Orden Operaciones en Talavera", dia 14 de septiembre de 1936. AGMA. Legajo 2/20 y D.N., O. M.,: «Orden de Operaciones particular para la Aviación (Ejército Expedicionario)», Cáceres, 14 de septiembre de 1936. AGMA. Legajo 2/21.2, p. 18. Sobre ello se pueden consultar también los partes de guerra del día 14-9-36: Partes ... Ejército Nacional, p. 38 y Partes ... Ejército de la República, pp. 5051. Declaraciones al autor confirmaron que ese día cayeron bombas en los domicilios particulares de "Cuquillo" e Hipólita. Testimonio de Lorenzo Orgaz Sánchez-Collado, El Casar de Escalona, julio 2005.

${ }_{18}$ La ejecución de la maniobra quedó perfectamente detallada en la "Orden de Operaciones dada en Talavera a 14 de Septiembre de 1936", firmada por el teniente coronel Juan Yagüe. Así consta en DNOM. AGMA. Legajo2/20. 
Varios días antes del 15 de septiembre llegó al pueblo un importante contingente de milicianos republicanos -en su gran mayoría varones pero también mujeres-que montaron su "cuartel general" en la Iglesia y sus alrededores y colocaron defensas basadas en ametralladoras en varios puntos estratégicos de la población (en la torre de la Iglesia, en la cuesta de Hormigos...). Hasta el día de los combates se limitaron a patrullar y no consta que hubiese incidente alguno con los vecinos, salvo peticiones de comida. Si causó malestar entre la población los destrozos que causaron en elementos y objetos del templo parroquial ${ }^{19}$.

\section{Día 15}

El encuentro del día 15 de septiembre frente al pueblo fue brutal, con un número indeterminado pero importante de bajas nacionales. Según llegaban los "moros" enrolados en los Regulares, procedentes de Castillo de Bayuela, eran repelidos una y otra vez. El combate se prolongó durante varias horas hasta que los republicanos, prácticamente rodeados, comenzaron a replegarse por las calles del pueblo. Los momentos de terror se sucedieron con persecuciones de milicianos, disparos y muertos. Todavía hoy los vecinos que lo vivieron -entonces niños a los que les sorprendió jugando- tienen grabadas las imágenes del «choque», la agonía de varios combatientes republicanos atrincherados en la torre de la Iglesia - previamente incendiada - que murieron tiroteados o se tiraron en llamas desde lo alto de la misma, los cadáveres dentro del casco urbano o amontonados a las afueras del pueblo de jóvenes, algunos agarrados - hombre y mujer-posiblemente como última reacción del que se sabe muerto... Por tanto, las bajas republicanas, aunque no podemos cuantificarlas, fueron también muy numerosas.

El municipio quedó prácticamente rodeado, sin embargo, los republicanos se replegaron hacia las encinas de La Dehesa y Los Lugares, puntos enclavados a varios kilómetros del centro, pero dentro de la jurisdicción. En la retirada hubo escaramuzas con cargas de bayonetas. Salvo error u omisión, ese día fallecieron once casareños por los disparos de los regulares que entraron en el pueblo. Se hizo salir a todos a la puerta de las casas y los momentos de terror se saldaron con el asesinato de al menos once personas, casi todos labradores y jornaleros agricolas. La entrada de las tropas moras fue bestial, con robos, violaciones y muertos, incluso alguno de ellos pereció a manos de sus propios mandos por actos de in-

19 En concreto, Lorenzo Orgaz refirió que los milicianos fueron por casa de sus padres y les confiscaron varios conejos que tuvieron que cocinarles. Testimonio de Lorenzo Orgaz Sánchez-Collado, El Casar de Escalona, julio 2005. Como analizamos en otro apartado, en la entrevista publicada en la revista nacionalista Horizontes, el alcalde de la villa en enero de 1938, Justo Acuña Arroyo, manifestó que espiritual y materialmente el pueblo quedó deshecho ante los hechos de la lglesia. Véase: Horizontes, "Casar de Escalona", n. 1 , enero de 1938. Artes Gráficas (Pasajes), Revistas Gráficas y Literarias, sin número. Los destrozos ocasionados a la Iglesia el 14-9-36 se denunciaron en el proceso judicial de la Causa General. Así quedó reflejado posteriormente en el Estado número 3. Archivo Histórico NacionalMadrid (AHN-M), FC-Causa General 1048-1, n. ${ }^{\circ} 3$. 
disciplina: el pillaje sustituía al combate por momentos (Cuadro n. $\left.{ }^{\circ}\right)^{20}$. En el avance del ejército de África se permitió por parte de sus mandos que, después de la ocupación de los pueblos, la tropa dispusiese de unas horas para ejercer su derecho de conquista. De esta forma, el día 15 de septiembre de 1936 El Casar sufrió en sus habitantes el "primer terror», la razzia después del combate en la que las tropas ocupantes obtenían su botín en bienes - robos en las casas de los vecinos-y "en sangre", como método eficaz para sujetar a la retaguardia civil. ${ }^{21}$ Respecto a los milicianos republicanos, presos de guerra, no poseemos datos numéricos concretos pero, por los testimonios consultados ${ }^{22}$, entendemos que ese día todos los que no escaparon del pueblo fallecieron. Además de los que murieron en los combates, muchos milicianos se escondieron en domicilios particulares, solicitando ser escondidos como hijos o familiares. En la mayoria de las ocasiones las marcas de los fusiles o de las mochilas les delataron; algunos también fueron denunciados. En cualquier caso, los nacionales fusilaron a un importante número de prisioneros.

Por lo tanto, una vez terminada la refriega los nacionales permanecieron horas en el lugar. De acuerdo con la documentación militar del ejército nacional, a las 21:00 horas del 15 de septiembre, se dio el municipio como ocupado y se instó a continuar el avance de las columnas de Castejón hacia el sur y el oeste para cortar la retirada de las fuerzas del gobierno. Sorprendentemente, el parte de guerra nacional del propio día 15 -información procedente del diario de las operaciones de las columnas de África - no hizo ninguna referencia a los acontecimientos y el republicano se centró en los avances del sector de Santa Olalla con la captura de "dos moros y un europeo con armamento". Una vez más, la información trasladada a la opinión pública resultó confusa y ocultista en función de los intereses ${ }^{23}$.

20 La brutalidad de estos momentos fueron revelados años atrás por familiares del autor, aunque afortunadamente solo tuvieron que lamentar robos en el domicilio. Testimonio de Ángeles Fernández Sánchez-Rico, El Casar de Escalona. En este sentido, el padre de Lorenzo Orgaz, guarda de profesión, estuvo a punto de morir como consecuencia de esta primera ocupación. Testimonio de Lorenzo Orgaz SánchezCollado, El Casar de Escalona, julio 2005; en la misma línea la entrevista con Gregorio Carretero. Testimonio de Gregorio Carretero Escobar, El Casar de Escalona, julio 2005. Los vecinos fallecidos ese día se registraron a posteriori. Para la relación de difuntos consúltese: "Certificaciones facultativas y demás justificantes de las defunciones cursadas durante el citado año" (1937 y 1939). Registro Civil de El Casar de Escalona. Archivo Municipal de El Casar de Escalona (AMECE). Otros datos proceden de Sección de Defunciones del Registro Civil de El Casar de Escalona, Libro 15 (9-11-35 al 10-7-47). AMECE.

21 Para un análisis más pormenorizado sobre el tema de la violencia y represión véase RUIz ALONSO, Op. Cit., II: pp. 335-337.

${ }_{22}$ En entrevista con el autor, Gregorio Carretero habló de muchos milicianos republicanos muertos. Testimonio de Gregorio Carretero Escobar, El Casar de Escalona, julio 2005.

${ }^{23}$ Sobre estos hechos revísese: DNOM: "Orden Operaciones en Cáceres", día 15 de septiembre de 1936. AGMA. Legajo 2/21.2, p. 26. Por otra parte, en los últimos días de septiembre son varias las referencias a la captura de "moros" en el sector Talavera-Santa Olalla. A este respecto examinar los partes de guerra del dia 15 de septiembre en Partes... Ejército Nacional, p. 38 y Partes ... Ejército de la República, pp. 51-52. Para un análisis más amplio, véase La Marcha sobre Madrid, Op. Cit., pp. 176177. 
Para Luis Moreno Nieto, cronista de la provincia de Toledo, aunque los combates no resolvieron nada en el aspecto militar sirvieron para la suspensión de los fusilamientos de cincuenta personas civiles previstos por los republicanos para ese día. No está demostrada la amenaza real de este hecho aunque parece verídico que algún mando miliciano amenazó con tomar represalias contra una serie de vecinos «derechistas" incluidos en una lista. No sabemos con seguridad si se trató de una medida disuasoria o si realmente la relación existió pero, una vez ocupada la población, se divulgó la noticia de su aparición en el local del Comité PopularCasa del Pueblo ${ }^{24}$. En cualquier caso, es un tanto extraño si tenemos en cuenta la escasez de documentos conservados en los locales del Frente Popular debido a su destrucción.

En todo caso, la versión optimista de que la «Columna Castejón» fue rechazada frente a El Casar y tuvo que retrasar la ocupación hasta el día 17 es bastante relativa. Los nacionales encontraron una feroz resistencia pero el pueblo se ocupó y lo cierto es que el ejército de África permaneció en el mismo hasta la madrugada del día 15 al 16, con todos los vecinos encerrados en sus casas, momento en que lo abandonó bien porque todavía no era un lugar seguro, bien por la falta de munición.

\section{Día 16}

En la documentación oficial hasta el día 16 de septiembre no apareció detallada la incursión a El Casar «que derrotó al ejército republicano y éste dejó abandonados en el interior doscientos muertos". No obstante, nuevamente el día 16 la columna que tenía encomendado el avance por el norte de la carretera general hasta el Alberche, siguiendo la directriz El Casar de Escalona-Hormigos-Maqueda, perseguía como primer objetivo: El Casar de Escalona-Otero-Domingo PérezErustes; esto suponía el reconocimiento implícito de que la villa no estaba todavía ocupada definitivamente ${ }^{25}$.

Ese día las milicias republicanas regresaron y se volvieron a vivir momentos de tensión en la localidad con acusaciones de "fascistas" contra los vecinos ante la falta de resistencia ofrecida a los soldados nacionales ("este pueblo es un pueblo fascista»). Algunos casareños se unieron ese día a las milicias de la República pero la mayoría abandonó el lugar aterrorizado por los sucesos del día anterior y lo que se avecinaba. Las decisiones de las familias variaron: unos se fueron a Ma-

24 Mírese la breve mención a los combates en Moreno NiETo, L., La provincia de Toledo. Toledo, Imprenta Diputación Provincial, 1960, p. 116. Los Comités Populares-Casas de Pueblo dispusieron de locales para sus actividades en casi todas las localidades toledanas. Para este menester en El Casar se utilizó un antiguo salón de baile junto a la tienda de "Balta».

25 DNOM: «Avance Nacional de Talavera a Maqueda del 3 al 21 ". AGMA. Legajo 2/18; igualmente los partes de guerra del día 16-9-36: Partes ... Ejército Nacional, p. 39 y Partes ... Ejército de la República, pp. 52-53. Hemos consultado también DNOM: «Orden Operaciones en Cáceres", dia 16 de septiembre de 1936. AGMA. Legajo 2/21.2, pp. 19-20. 
drid, otros se escondieron en el campo, algunos huyeron en dirección a Hormigos para ocultarse en casa de parientes, incluso pasaron a la otra margen del río... De uno $u$ otro modo, la población quedó prácticamente vacía. Todo indica que la jornada del día 16 fue muy complicada, con importantes bombardeos y contraataques de la resistencia republicana por la comarca. El desenlace negativo de las operaciones para el ejército nacional obligó a intervenir a las unidades de Asensio y a las de Castejón que bajaron de la Sierra de San Vicente.

\section{Día 17}

Ante tal acumulo de fuerzas nacionalistas, por fin, el 17 de septiembre de 1936 alcanzaron la línea de El Casar de Escalona-El Bravo-Los Cerralbos-llián de Vacas. El diario de operaciones del ejército nacional reflejó la ocupación de estos pueblos y la huida de los «rojos» dejando 538 muentos. El bando republicano no reconoció - de forma oficial- los nuevos avances nacionales ${ }^{26}$.

Los combates decisivos del día 17 fueron en "La Carrasca", finca situada a varios Kilómetros del casco urbano de El Casar, en dirección a Talavera, junto al camino de El Bravo. Con la República participó la Guardia de Asalto, verdadero cuerpo de elite leal al gobierno. La batalla, otra vez muy fuerte, causó también numerosas pérdidas humanas - principalmente republicanas- y ese día se rompió definitivamente la segunda línea de contención del eje Cazalegas-Maqueda.

\section{Dias 18,19 y siguientes}

Finalmente, entre el 18 y el 19 de septiembre, la mayoría de los lugareños que huyeron aterrorizados volvieron a sus casas (con banderas blancas). Los que lo hicieron a Madrid o al sur del Tajo ya no regresarían hasta el final de la guerra, no obstante, el temor no había pasado todavía, ahora, intentando recuperar posiciones, la aviación republicana bombardeó el pueblo durante varias horas -cayeron más de treinta bombas- afortunadamente sin muertos. El día 18 Asensio ocupó Otero.

La ordenada resistencia republicana retrasó la ocupación definitiva de El Casar de Escalona y todas las operaciones programadas, por ello, el primer Tabor de Regulares de Tetuán, que participó en su "conquista" hubo de esperar para reforzar a la Columna de Barrón —ubicada en El Bravo- hasta el relevo del puesto por el

26 DNOM: "Avance Nacional de Talavera a Maqueda del 3 al 21 (Varias columnas)". AGMA. Legajo 2/18. Utilizamos también como fuente La Marcha sobre Madrid, Op. Cit., pp. 176-177; Véase finalmente para este punto los partes de guerra del día 17-9-36: Partes ... Ejército Nacional, p. 39 y Partes ... Ejército de la República, pp. 53-54. 
Tabor de Regulares de Melilla. Como podemos apreciar, las unidades cambiaban a una u otra columna o agrupación, según las conveniencias tácticas ${ }^{27}$.

El paso absoluto del pueblo a la zona nacional tuvo su plasmación inmediata en la sustitución de los miembros de la Corporación municipal. Al igual que en otras localidades, lo primero que hicieron los militares consistió en designar una Comisión Gestora provisional de entre los vecinos más fiables para el nuevo régimen (falangistas, propietarios que habían sufrido represión en el periodo anterior, etc.). A tal efecto, el Ayuntamiento celebró una importante sesión extraordinaria el dia 19 de septiembre de 1936, presidida por un capitán -en nombre del teniente coronel del Grupo de Regulares de Melilla Fernando Barrón Ortiz- en la que se eligió a la nueva Junta, que quedó integrada por: Isidro Medina Toro como Presidente y por Eduardo Palomo Guadalupe, Pablo Escobar Murcia, Julián Arroyo Madrid, Jesús Escobar Valencia, Guillermo Valencia Fernández y Eladio Loarce López, como vocales. Días después, ya como alcalde Isidro Medina, fueron designados Eduardo Palomo y Pablo Escobar para primer y segundo teniente de alcalde respectivamente y Jesús Escobar, como regidor síndico ${ }^{28}$.

En resumen, El Casar de Escalona se encontró en el camino de la "Columna Madrid" y por azares del destino fue uno de los puntos elegidos por el gobierno de la República como línea defensiva. El «fatídico» martes 15 de septiembre la Columna de Castejón procedente de Castillo de Bayuela-Cardiel de los Montes no consiguió totalmente su objetivo, sufrió fuertes bajas no cuantificadas -los testimonios hablan del elevado número de "tropas moras" que perdieron la vida en el choque- y ante la situación, en la madrugada del 15 al 16 se replegó. Tan duros fueron los combates y la resistencia encontrada que las tropas nacionales tuvieron que esperar refuerzos y demoraron hasta el día 17 de septiembre la toma definitiva de la villa.

La llegada de los milicianos, la entrada de los "moros" con los regulares "arrasando" y matando, la violencia y los bombardeos de esos días marcaron por mucho tiempo la vida de estos pueblos, con episodios de represión masiva constatados en algunos y con muchos indicios en otros. El Casar pasó de este modo en momento temprano al territorio nacional y, por su situación geográfica, la localidad quedó dependiente administrativamente de Toledo junto con el resto de municipios al norte del río Tajo (la zona leal a la República estableció su capital en Ocaña).

\subsection{La ruptura definitiva del sector}

El último elemento del repliegue, y núcleo principal del dispositivo defensivo, se situó en Santa Olalla-Maqueda. Los partes militares del 18 y 19 de septiembre no

27 DNOM: «Partes de Operaciones y relaciones de bajas cursadas al Jefe de la Columna Madrid, General Yagüe», El Bravo, 17 de septiembre de 1936. AGMA. Legajo 3/2.1, p. 29.

${ }_{28}$ Todo ello consta en el Libro de Actas del Ayuntamiento, sesión del 19-9-36. AMECE, p. 59. Para más información sobre este tema consúltese también la sesión del 30-9-36. AMECE, p. 60. 
trasladaron a la opinión pública ninguna información clarificadora de los movimientos militares. De cualquier modo, independientemente de la información oficial, el día 19 la Columna de Barrón se encontraba desplegada en El Casar de EscaIona, la de Castejón en El Bravo y la de Asensio en Otero, cada una compuesta por tres unidades y dos baterías y se estaba preparando el ataque contra Santa Olalla. Para la conquista de esta última población se pretendía que Barrón la envolviese por el norte y que Castejón atacase de frente para que Asensio, con vía libre, ocupase la localidad ${ }^{29}$. Finalmente, las milicias republicanas - al mando del general Asensio Torrado - no pudieron contener al enemigo y Santa Olalla fue tomada el día 20. Los partes de guerra se mostraron - como siempre-contradictorios, el nacional refería la ocupación de Santa Olalla con la captura de numerosos prisioneros y material (con muchos muertos abandonados en el campo), sin embargo, el republicano solamente reconocía el violento ataque sufrido en el sector ${ }^{30}$.

En su táctica militar de afianzar las posiciones, el 20 de septiembre quedó la cuarta Bandera del ejército nacional en El Bravo, una compañia de Alhucemas en El Casar de Escalona y otra en Otero. Para la invasión de Maqueda, de acuerdo con lo previsto, se siguió la carretera general y se ocupó el dia 21 de septiembre "por el único sitio no fortificado". Desde Santa Olalla se acometió igualmente la ocupación de Val de Santo Domingo, hecho consumado por Asensio - no confundir con Asensio Torrado- el día 22. Por otra parte, en una acción conjunta de Castejón, marchando desde Alcabón con Asensio por el norte, Torrijos "cayó" también el día 22 de septiembre. Por supuesto, los partes de guerra del ejército nacional se hacían eco de estas importantes victorias, incluso en el caso de la localidad de Torrijos ese día se dieron cifras redondas de bajas: "...después de derrotar al enemigo que dejó en poder de las tropas ochenta muertos y algunos prisioneros". Paradójicamente, cuando ya se encontraba totalmente rota la línea defensiva Cazalegas-Maqueda, en el parte republicano por primera vez se «mejoran posiciones" en el sector Talavera-Santa Olalla. A partir del día 25 de septiembre los partes de guerra nacionales se centraron en el avance de las columnas hacia Toledo ${ }^{31}$.

A pesar de los progresos y el afianzamiento de las posiciones nacionalistas, la conflictividad de estas comarcas continuó y pueblos cercanos a El Casar, sin trascendencia, conquistados en septiembre, como Pelahustán -el día 12 concre-

${ }^{29}$ DNOM: “Orden de operaciones en Talavera", 19 de septiembre de 1936. AGMA. Legajo 2/20. En este punto hemos seguido también La Marcha sobre Madrid, Op. Cit., p. 177 y los partes de guerra de los días 18 y 19-9-36: Partes ... Ejército Nacional, p. 40 y Partes ... Ejército de la República, pp. 54-56.

${ }^{30}$ Los datos proceden de los partes del 20-9-36: Partes ... Ejército Nacional, p. 40 y Partes ... Ejército de la República, pp. 56-57; y de la fuente original conservada en D.N., O. M.,: “Operaciones del avance Nacional de Talavera a Maqueda del 3 al 21 (Varias Columnas)", 20 de septiembre de 1936. AGMA. Legajo 2/18.

31 Todo ello se puede consultar más ampliamente en DNOM: "Orden de operaciones en Talavera", 21 de septiembre de 1936. AGMA. Legajo 2/20; Véase también los partes de guerra de esos días: Partes ... Ejército Nacional, p. 41 y Partes ... Ejército de la República, pp. 58-60; igualmente en La Marcha sobre Madrid, Op. Cit., p. 177. 
tamente- tuvieron que ser retomados en octubre -el día 11-debido a la resistencia local y a la efectividad de las columnas republicanas, que formaron una nueva línea defensiva, con obras de fortificación en torno a Escalona. Hasta ese momento se había ocupado una estrecha franja de terreno desde Talavera a Maqueda no exenta de indefensión (tenía uno de sus flancos apoyado en el río y el otro en las estribaciones de la Sierra de San Vicente). Por ello, ante la precariedad del dominio, el general Franco decidió, el 30 de septiembre, situar en los puntos principales - El Real de San Vicente, Cazalegas, El Casar de Escalona, Santa Olalla, Maqueda, Torrijos, etc.- a una serie de compañías del Regimiento de Argel, de carabineros, de voluntarios de Cáceres, Sevilla, Canarias y alguna unidad del propio Ejército Expedicionario. Así, el ejército nacional cubría su retaguardia. ${ }^{32}$ El Casar quedó como un lugar estratégico en la marcha hacia Madrid, como una posición militar entre el río Tiétar y la ciudad de Toledo.

En suma, de acuerdo con los hechos analizados, el ejército africano sufrió numerosas pérdidas en esta fase de la guerra y tardaron nada menos que once dias para recorrer los $33 \mathrm{Km}$. de distancia entre Cazalegas y Maqueda, a pesar de una superioridad manifiesta en efectivos y medios. Pero la resistencia tuvo un límite y la ocupación de Santa Olalla, Maqueda y Torrijos en tres días fue un golpe muy duro para el gobierno de Largo Caballero que además provocó numerosas deserciones de milicianos. Lo ocurrido en la Sierra de San Vicente - Pelahustán se ocupó dos veces en un margen corto de tiempo- y El Casar de Escalona -el ejército nacional tuvo que replegarse y diferir la ocupación definitiva del 15 al 17 de septiembre - no fue casual. Dentro de sus limitaciones y su inexperiencia, la milicia republicana intentó combatir, se opuso al avance y cambió momentáneamente la dinámica bélica hasta ese momento. Por su parte, el ejército de África tomó las poblaciones de forma muy violenta y causando importantes estragos, sobre todo en la vecina Santa Olalla.

\subsection{La prensa y las operaciones militares (del 12 al 22 de septiembre)}

La moderación informativa de la prensa republicana leal (El Liberal, Heraldo de Madrid, El Sol, $A B C$-en su edición madrileña- etc.), presidió la narración de las operaciones militares durante los días en que se gestó la ocupación de El Casar de Escalona y la ruptura definitiva de su eje defensivo. En general, en estos días vitales para el sector, la prensa ocultó información - suponemos que por la censura previa-y se limitó, en muchas ocasiones, a la trascripción de los partes de guerra republicanos. Todos los titulares mantenían una línea triunfalista ante la guerra, independientemente de la marcha real de esta. ${ }^{33}$ Para contrastar y complementar los

32 La Marcha sobre Madrid, Op. Cit., p. 214.

33 $\mathrm{Si}$ se quiere ampliar información sobre la prensa toledana durante la Guerra civil léase MIRANDA ENCINAS, J. M., La prensa en la Guerra Civil. Toledo: 1936-1939. Toledo, Diputación Provincial de Toledo, 2004. Hemos consultado varios números de prensa republicana de esos días, entre otros, resultan de in- 
movimientos militares del tramo Cazalegas-Maqueda, se antoja imprescindible la consulta de un diario nacionalista como El Alcázar-diario del Asedio- cuyo primer ejemplar apareció el 26 de julio y el último el 27 de septiembre de 1936. Podemos considerar este periódico como una excepción de prensa de la zona nacional de alto valor histórico, a diferencia de otras publicaciones posteriores dirigidas a los combatientes. Para que nos hagamos una idea de su visión optimista del conflicto, el día 15, tras captar emisiones republicanas, el redactor de El Alcázar deducía que los nacionales estaban ya cercanos a Santa Olalla ${ }^{34}$.

En esta línea triunfalista, casi épica, destacamos también las numerosas publicaciones periódicas nacionales sobre los acontecimientos ocurridos en septiembre del 36. Un artículo dedicado a la ocupación de El Casar, incluido en un número de la revista Horizontes sobre la provincia de Toledo ${ }^{35}$, partía de la llegada de los «milicianos faístas» para preparar la defensa del lugar, pasando por los sucesos del día 15, cuando las tropas de Castejón «siembran el pánico en la chusma roja», hasta que, finalmente, los legionarios de Barrón tomaron el pueblo el día 17 y lo incorporaron definitivamente a la «nueva España».

\subsection{Franco alcanza la jefatura del Estado}

De acuerdo con Rafael Abella ${ }^{36}$, el mes de septiembre resultó favorable para el ejército nacionalista. Después de tomar Irún y San Sebastián se inició el avance por la provincia de Toledo y en Gredos contactaron las tropas del ejército del Norte y las del Sur, mandadas por Monasterio y Tella respectivamente. Se apoderaron, no sin problemas, de Talavera, Cazalegas, El Casar de Escalona, Santa Olalla, Maqueda, Torrijos, etc. La marcha hacia Madrid era recta pero, una decisión histórica - una vez arrebatada Maqueda el 21 de septiembre de 1936- llevó a Franco a ordenar el desvío hacia Toledo, en defensa del Alcázar, resolución muy criticada por todos los especialistas militares. Los ojos del mundo se fijaron en Toledo y el sitio del Alcázar. Hasta tal punto resultó trascendente su conquista que, solo tres días después la Junta de Defensa Nacional reunida en Salamanca, nombró a Franco Jefe del Estado.

El cambio de Yagüe por Varela, efectivo el 22 de septiembre, parece íntimamente relacionado con la decisión de encaminarse hacia Toledo. Para Yagüe, la

terés para nuestro estudio: Heraldo de Madrid, 15-9-36, p. 2; Idem, 16-9-36, p. 2; Idem, 17-9-36, portada; Idem, 18-9-36, portada y p. 3; Idem, 21-9-36, portada; Idem, 22-9-36, portada. El Sol, 15-9-36, p. 3; Idem, 16-9-36, portada; Idem, 17-9-36, portada; Idem, 18-9-36, portada; Idem 19-9-36, portada. El Liberal, 16-9-36; p. 3; Idem, 19-9-36, p. 3. ABC (madrileño), 18-9-36, p. 9.

${ }_{34}$ Una información más pormenorizada puede verse en Miranda EnCINAS, J.M., Op. Cit., pp. 71-75. Mírese sobre el particular El Alcázar (Asedio), n. ${ }^{\circ} 48,12-9-36, p .3$; Idem, n. ${ }^{\circ}$ 51, 15-9-36, p. 1; Idem, n. ${ }^{\circ}$ 52, 16-9-36, p. 2; Idem, n. ${ }^{\circ} 53,17-9-36$, pp. 2-3; Idem, n. ${ }^{\circ} 57,21-9-36$, pp. 1-2.

${ }_{35}$ Horizontes, Op. Cit.

36 Abella, R., La vida cotidiana durante la Guerra Civil. La España Nacional. Barcelona, Editorial Planeta, 2004, pp. 67 y 76-77. 
«liberación» de Madrid seria más dificil cuanto más se retrasase la operación y militarmente era un rodeo poco o nada afortunado. Sin embargo, Franco le relevó y puso al mando al general José Enrique Varela, apoyado por Asensio y Barrón. El general Varela reorganizó las columnas a su mando y dejó además una fuerte reserva de tropas en otras localidades como El Casar de Escalona ${ }^{37}$.

En definitiva, independientemente de que fuese o no un grave error militar, el desvio hacia Toledo, una vez tomada Maqueda, su conquista y, en general, los éxitos militares de septiembre auparon a Franco a la Jefatura del Estado. Los acontecimientos forzaron el relevo de Yagüe por Varela.

\section{CONCLUSIONES}

Ante el fracaso de la insurrección como golpe militar comenzaron los primeros combates. Las masas querían luchar y lo hicieron con heroicidad, se dio un alto grado de movilización de la población que cristalizó en las milicias -en los dos bandos- y en una guerra de guerrillas primitiva, casi ancestral. Con el paso del ejército a la península - gracias a la ayuda alemana e italiana - los nacionales consiguieron inclinar la balanza de su lado. El hecho, dada su trascendencia, se ocultó por las autoridades republicanas el mayor tiempo posible. Ya en la península, la denominada "Columna Madrid» realizó un itinerario pegado a la frontera portuguesa y con el apoyo del régimen del país vecino, de Alemania y de Italia, llegó a Sevilla el 7 de agosto. Por consiguiente, desde el 28 de julio de 1936 comenzó la fase dinámica de la guerra, la única que, a pesar de los rasgos un tanto atrasados, funcionó como campaña relámpago a través de Andalucía, Extremadura -escasamente poblada - y el valle del Tajo sin baluartes defensivos.

La caída de Talavera de la Reina desencadenó consecuencias políticas fulminantes para el gobierno republicano, con dimisiones incluidas. Largo Caballero, jefe del Gobierno, ordenó al militar leal Asensio Torrado poner orden en el frente y este sorprendió al ejército africano con una serie de líneas defensivas escalonadas, basadas en fortificaciones y trincheras en torno a la carretera general y un importantisimo contingente de hombres dispuestos a combatir pero, cometió un error clave, y una gran oportunidad de romper el frente nacionalista, al enviar tropas para conquistar el Alcázar de Toledo. Esto fue aprovechado por los nacionales para la «liberar» Santa Olalla y luego Maqueda. El Casar de Escalona se encontró en el

${ }^{37}$ SABIN, J. M., Op. Cit., 143; Varela organizó tanto la composición de las columnas como la regulación de sus servicios: Municionamiento, Ingenieros, Sanidad, Correos, Automovilismo, etc. Cuatro columnas numeradas del 1 al 4 y mandadas por los tenientes coroneles Asensio, Barrón y Delgado Serrano y comandante Castejón, además de la columna de Caballería del coronel Monasterio. En El Casar quedó: «P, M, 2. ${ }^{\circ}$ Tabor de Regulares de Tetuán, $1 \mathrm{Cia}$. de fusiles de idem idem, 1 Centuria de Falange de Canarias". Para revisar la reorganización completa examínese DNOM: "Ordenes de Organización. Grupo de columnas del general Varela", Toledo, 29 de septiembre de 1936. AGMA. Legajo 3/1.1. Sobre este respecto consúltese además La Marcha sobre Madrid, Op. Cit., pp. 214-215. 
camino de la "Columna Madrid» y por azares del destino resultó uno de los puntos elegidos por el gobierno de la República como línea de contención. En la fatídica jornada del 15 de septiembre - que hemos denominado el "martes sangriento" por su violencia y número de víctimas - la Columna de Castejón encontró fuerte resistencia frente a El Casar y sufrió numerosas bajas, principalmente entre sus tropas moras. Ante las noticias de movimientos de milicias republicanas en la madrugada del 15 al 16, en espera de aumentar sus efectivos, se replegó. Finalmente, el 17 de septiembre, después de los combates de "La Carrasca", ocuparon definitivamente la localidad.

En suma, el ejercito de Marruecos perdió numerosos hombres en esta fase de la guerra y tardó nada menos que once días en recorrer los 33 kilómetros de distancia entre Cazalegas y Maqueda, a pesar de su superioridad en efectivos y medios. Pero la resistencia tenía su límite y la ocupación de Santa Olalla, Maqueda y Torrijos en tres días fue un golpe muy duro para el gobierno que desmoralizó a las tropas y provocó numerosas deserciones. Sin embargo, los episodios de la Sierra de San Vicente (Pelahustán) y El Casar de Escalona no fueron casuales. EI ejército republicano se opuso al avance y cambió momentáneamente la dinámica de la guerra civil. Por su parte, los componentes del ejército de África, curtidos en el Rif, actuaron de forma muy violenta y causaron importantes daños en las localidades ocupadas. Por último, el desvío hacia Toledo una vez tomada Maqueda -considerado por todos un grave error militar - su conquista y, en general, los éxitos militares de septiembre, llevaron a Franco a la Jefatura del Estado.

Este trabajo no pretende reabrir viejas heridas, todo lo contrario, pero el día 15 de septiembre -con once muertos solo entre casareños- pasará a la posteridad como uno de los más violentos, sin parangón en la historia de El Casar desde su pasado medieval, como aldea fronteriza de la tierra de Escalona. 